\title{
Análise dos indicadores de ciência, tecnologia e inovação no âmbito da Fundação Oswaldo Cruz (Fiocruz)
}

Pierre Ohayon

Universidade Federal do Rio de Janeiro (UFRJ)

Gerson Rosenberg

Fundação Oswaldo Cruz (Fiocruz/MS)

Os tomadores de decisão reconhecem que é necessário não só dispor de uma base de dados confiável e efetiva sobre ciência, tecnologia e inovação (CT\&I), como também dispor de um modelo de indicadores capaz de dar conta da difícil tarefa de bem avaliar a aplicação dos recursos públicos. O estudo objetivou a formulação de indicadores de CT\&I, de forma a contemplar a abordagem holística de uma instituição de pesquisa na área da saúde, a Fundação Oswaldo Cruz (Fiocruz). Levantaram-se os indicadores encontrados principalmente nos relatórios de gestão da Fiocruz, confrontando-os com o modelo conceitual. Como resultado dessa análise comparativa, verificou-se a presença de indicadores com algumas limitações e a falta de indicadores relevantes. Apontaram-se outros indicadores visando a cobrir todas as dimensões e subdimensões do modelo proposto. Sugeriram-se recomendações para que o modelo esteja consistente com as políticas do Ministério da Saúde do Brasil.

Palavras-chave: ciência e tecnologia, inovação, indicador, avaliação de programas, administração pública

\section{Análisis de los Indicadores de Ciencia, Tecnología e Innovación en la Fundación Oswaldo Cruz (Fiocruz)}

Los tomadores de decisiones reconocen la necesidad de no sólo tener una base de datos confiable y eficaz de ciencia, tecnología e innovación (CT\&l), sino también de tener un modelo de indicadores capaces de hacer frente a la difícil tarea de evaluar mejor la aplicación de los recursos públicos. El estudio tuvo como objetivo formular indicadores de

Artigo recebido em outubro de 2013. Versão final em junho de 2014. 
CT\&I con el fin de tener en cuenta el enfoque holístico de una institución de investigación en el área de la salud, la Fundación Oswaldo Cruz (Fiocruz). Se plantearon indicadores encontrados principalmente en los informes de la gestión de la Fiocruz, confrontándolos con el modelo conceptual. Como resultado del análisis comparativo se ha verificado la presencia de indicadores con algunas limitaciones y la falta de indicadores pertinentes. Señaló a otros indicadores con el fin de cubrir todas las dimensiones y subdimensiones del modelo propuesto. Se sugieren recomendaciones para que el modelo se muestre coherente con las políticas del Ministerio de la Salud de Brasil.

Palabras clave: ciencia y tecnología, innovación, indicador, evaluación de programas, administración pública

\section{Science, Technology and Innovation Indicators' Analysis within the Oswaldo Cruz Foundation - Fiocruz (Brazil)}

Decision makers recognize the need to have not only a reliable and effective database on Science, Technology and Innovation (ST\&I), but also a model of indicators able to cope with the difficult task of better evaluating the application of public resources. The objective of this study was to formulate ST\&I indicators in order to consider a holistic approach of a research institution in healthcare, the Oswaldo Cruz Foundation - Fiocruz. Indicators found mainly in Fiocruz's management reports were pointed out and compared with a conceptual model. As a result of this comparative analysis, the presence of limited indicators has been verified as well as the lack of relevant ones. Other indicators were pointed out in order to cover all dimensions and sub-dimensions of the proposed model. Recommendations were suggested for the model to be consistent with the Brazilian Ministry of Health policies.

Keywords: science and technology, innovation, indicator, program evaluation, public administration 


\section{Introdução}

Os impactos da tecnologia no desenvolvimento econômico já há muito são objeto de estudo de organizações como a Organização para a Cooperação e Desenvolvimento Econômico (OCDE), a Organização das Nações Unidas para a Educação, a Ciência e a Cultura (Unesco) e a Red de Indicadores de Ciencia y Tecnología Iberoamericana (RICYT). A preocupação já deixou de ser discussão acadêmica para inserir-se nas preocupações de governos nacionais e estaduais, a fim de lidar com as transformações geradas no mercado de trabalho, mercado consumidor, na infraestrutura, na educação e no meio ambiente (OHAYON, 1991, BRISOLLA, 1998, LIBERAL, 2005).

Viotti e Macedo (2003), Liberal (2005) e Brisolla (1999) apontam que há muitos estudos sobre indicadores científicos e tecnológicos no Brasil, sobretudo a partir de 1990, que resultaram em várias publicações tanto em nível nacional quanto estadual, a exemplo dos "Indicadores de Ciência, Tecnologia e Inovação em São Paulo, 2010", publicação realizada pela Fundação de Amparo à Pesquisa do Estado de São Paulo (FAPESP, 2010). No Rio de Janeiro, pode-se apontar o estudo realizado por Ohayon (2007), que consistiu na elaboração de um modelo de indicadores voltado para as atividades da Fundação de Amparo à Pesquisa do Estado do Rio de Janeiro (FAPERJ).

A construção de indicadores se justifica, pois, de fato, vive-se, em relação a outros períodos da história, uma pujança de recursos humanos, materiais e financeiros direcionados para o desenvolvimento de CT\&I (BERRY e TAGGART, 1994, p. 343). Portanto, o problema em questão passa a ser o melhor gerenciamento desses recursos e, para esse propósito, a avaliação torna-se útil como instrumento de planejamento, de direção e de controle da gestão de ciência, tecnologia e inovação. Segundo Ohayon (2007), o estudo dos indicadores é importante para toda avaliação e análise estratégica que se faça nas atividades de ciência e tecnologia. Eles auxiliam a gestão, pois demonstram a relação e o grau de eficiência com que os recursos financeiros, materiais e humanos alocados (inputs) produzem o resultado (outputs).

O presente estudo tem como objetivo geral identificar os indicadores de CT\&I utilizados por uma organização pública de pesquisa, a Fundação Oswaldo Cruz (Fiocruz), com a finalidade de avaliar o seu desempenho institucional, e comparálos com o modelo de avaliação proposto por Ohayon (2007), sugerindo outras dimensões avaliativas, a fim de contribuir para a solidez do sistema avaliativo e torná-lo mais eficaz no tocante ao controle do cumprimento da missão institucional. 


\section{Referencial conceitual e o modelo proposto}

As instituições de CT\&I, colocadas num ambiente caracterizado por fortes turbulências e incertezas crescentes, necessitam dotar-se de uma abordagem prospectiva e estratégica de suas ações. Assim, para cada ação estabelecida é preciso que se identifiquem as forças e fraquezas internas em relação aos objetivos de longo prazo, bem como as ameaças e oportunidades externas. Não causa surpresa que, nessas condições, a Análise Estratégica e Prospectiva (AEP) assim como a avaliação sejam cada vez mais consideradas pela comunidade científica e técnica como funções essenciais para a adequada aplicação dos recursos públicos ou mesmo privados. Entretanto, a AEP, tanto quanto a avaliação, pode ser efetuada somente se houver indicadores pertinentes e suficientemente confiáveis (BARRÉ, 1997).

Segundo Hadji (1994), a avaliação é

o ato pelo qual se formula um juízo de valor, incidindo num objeto determinado (indivíduo, situação, ação, projeto etc), por meio de um confronto entre duas séries de dados que são postos em relação: (i) dados que são da ordem do fato em si e que dizem respeito ao objeto real a avaliar; (ii) dados que são da ordem do ideal e que dizem respeito a expectativas, intenções ou a projetos que se aplicam ao mesmo objeto (HADJl, 1994, p. 31).

Assim, no conceito da avaliação estão inseridos três elementos importantes que são: o ato da medição e do controle; a congruência entre o desempenho e os objetivos; e o processo de julgamento por um profissional. Pode-se também entender a avaliação como uma "coleção sistemática de informações acerca de atividades, características e resultados (outcomes) de ações para uso de pessoas específicas com a finalidade de reduzir incertezas, melhorar a efetividade e tomar decisões, em relação ao que essas ações estão fazendo e influenciando" (CLARK, 1999, p. 14 apud LIMA, 2004). Nesse contexto, é importante avaliar a própria avaliação como sistema de auxílio à decisão (ContANDriopoulos, 2011, p. 264).

A necessidade de avaliação dos resultados decorrentes das ações de pesquisa científica, desenvolvimento tecnológico e inovação vem se manifestando crescentemente, e, portanto, a avaliação deixa de ser um exercício puramente técnico, transformando-se progressivamente numa vontade política. Os responsáveis por essas ações em CT\&l estão empenhados em exercer escolhas e definir estratégias. Nesse sentido, as agências de fomento, assim como as organizações sociais tais como o Centro de Gestão e Estudos Estratégicos (CGEE), vêm induzindo, de um lado, o desenvolvimento da prospectiva e, de outro, a atribuição à avaliação de um papel-chave. Trata-se de integrar o processo de avaliação ao processo decisório. Isso é, criar uma nova maneira de gerenciar a 
CT\&I que, a partir do exame dos resultados alcançados, identifica as orientações temáticas/estruturais ou ações pontuais a serem privilegiadas. É o que se poderá chamar de aspectos "transversais" da avaliação. Rompe-se com a prática antiga de se limitar aos aspectos "verticais" da avaliação (essencialmente técnicocientíficos), alargando-a aos aspectos "transversais" (qualidade dos resultados, efeitos socioeconômicos, qualidade da gestão).

Assim, a avaliação tende a sair do terreno estritamente científico para penetrar no terreno da política. Quase três décadas se passaram desde as primeiras experiências de avaliação de programas governamentais no Brasil e, apesar da importância de que o assunto se reveste, o avanço em relação ao desenvolvimento e implantação de sistemas de avaliação de CT\&I não foi muito significativo.

O papel da avaliação vem ganhando importância como instrumento de gestão, especialmente para a tomada de decisão, ou seja, para o estabelecimento das políticas públicas, ao mesmo tempo em que a administração pública sofre transformações causadas pela descentralização gerencial, o que dilui as responsabilidades dos gestores, impedindo o desenvolvimento de mecanismos de integração (SILVA E SILVA, 2001, p. 13). É cada vez mais valorizado e ressaltado, no mundo desenvolvido, o caráter sistêmico das inovações, sobretudo a importância da integração das atividades de C\&T e dos agentes com os sistemas produtivos locais e regionais de inovação, de modo a prover desenvolvimento econômico e social mais sustentável (WORTHEN et al., 2004).

Construir indicadores e sistemas de avaliação é, portanto, condição prévia para o estabelecimento de políticas orientadas para a promoção da inovação tecnológica. A avaliação pode ser um meio para integrar a realidade complexa das relações entre os Poderes públicos (em âmbito local, regional e nacional), sistemas produtivos e sociedade civil; essa última, real financiadora e objeto dos programas engajados. As avaliações de programas focalizam aspectos tais como: critérios de eficiência, eficácia, efetividade e qualidade da gestão. É preciso destacar também que a produção de indicadores não é a avaliação, mas lhe é necessária. Dessa forma, os indicadores de CT\&I analisados de forma sistêmica por meio de um modelo permitem possíveis formulações globais sobre o próprio sistema de indicadores existente da Fiocruz e sobre os processos que o caracterizam. Por meio dos indicadores, buscam-se medir, em particular, os graus de eficiência e de eficácia, visando a tornar coerentes os resultados com os objetivos básicos da instituição. Esses indicadores devem ser entendidos como instrumento de avaliação e não de controle, pois não visam a ações corretivas imediatas.

O modelo proposto no Quadro 1 é composto de seis dimensões avaliativas, a saber: recursos (inputs); dinâmica das atividades de CT\&I (processo); resultados 
diretos (outputs diretos); atividades de produção científica e técnica na Fiocruz (outputs indiretos); utilização dos resultados; e efeitos. São apresentadas para cada dimensão subdivisões de modo a especificar os aspectos avaliados e os respectivos indicadores integrantes. Cada subdimensão possui um número de indicadores específicos, de modo que esses sejam relevantes para a tomada de decisão e confiáveis quanto à sua medição. Por exemplo, na dimensão dos indicadores de inputs destaca-se uma subdimensão própria para tratar de recursos financeiros aportados para a CT\&I na Fiocruz.

Praticamente cada subdimensão é constituída pela representação de diferentes indicadores, quantitativos ou não, sob formas diversas (quadros, gráficos) acompanhadas de comentários, evidenciando os pontos essenciais, assim como os limites da interpretação. Tal representação deve ser complementada por definições, explicações metodológicas e bibliografia. Esse conjunto de indicadores permite avaliar a gestão de CT\&I.

A organização dos indicadores em dimensões e subdimensões, bem como a análise por intermédio de um sistema integrado permitem inferências sobre o organismo em observação referentes a aspectos como: subsistemas, interdependências, limites e fronteiras, centralização e descentralização, entre outros aspectos. Assim, o modelo expressa o conceito de sistema, adequado às atividades de CT\&I (OHAYON, 2007). Respeitaram-se as dimensões e as definições apresentadas no modelo conceitual; porém, para algumas subdimensões, foram necessárias revisões a fim de adaptá-las às atividades desenvolvidas pela Fiocruz. Para tanto, substituíram-se os resultados diretos e indiretos apresentados.

O modelo sugerido para a Fiocruz tem utilidade tanto para a avaliação ex-ante quanto ex-post, ou mesmo acompanhamento. A primeira, como avaliação proativa, é voltada para o futuro, correspondendo à denominada análise estratégica. Ela visa a lidar com uma nova situação, definindo uma estratégia como sendo uma função de forças e fraquezas internas e ameaças e oportunidades externas. A segunda, como avaliação retroativa, que lida com o passado, visa a situar resultados alcançados em relação a objetivos declarados, assim como examinar as condições para a implementação dos procedimentos necessários para o alcance dos mesmos. Inclui geralmente recomendações para o futuro. Entre essas duas avaliações, há o acompanhamento ou mesmo progresso das atividades de CT\&I da instituição (RUBENSTEIn, 2001, p. 309). Uma equipe é organizada para implementação dessa responsabilidade. O Quadro 1 apresenta o modelo adaptado de dimensões e subdimensões de indicadores de CT\&I. 


\section{Quadro 1: Dimensões e Subdimensões de Indicadores de CT\&I}

\begin{tabular}{|l|l|}
\hline Dimensões & $\begin{array}{l}\text { Subdimensões } \\
\text { Recursos Humanos/Recursos Financeiros/ } \\
\text { Recursos Materiais e Espaço Físico/Recursos } \\
\text { Informacionais/Recursos Organizacionais }\end{array}$ \\
\hline $\begin{array}{l}\text { Dinâmica das Atividades de CT\&I } \\
\text { (processo) }\end{array}$ & Gestão/Cooperação e Abertura/Estratégia \\
\hline Resultados Diretos (outputs diretos) & Pesquisa/ Educação/ Difusão \\
\hline $\begin{array}{l}\text { Atividades de Produção Científica e } \\
\text { Técnica (outputs indiretos) }\end{array}$ & $\begin{array}{l}\text { Projetos, Programas e Ações Desenvolvidas/ } \\
\text { Publicações de Artigos e Teses/Orientação de } \\
\text { Teses }\end{array}$ \\
\hline Utilização dos Resultados & $\begin{array}{l}\text { Para Evolução da Ciência: Mobilidade Temática/ } \\
\text { Para Desenvolvimento Tecnológico/Para Comer- } \\
\text { cialização }\end{array}$ \\
\hline Efeitos & $\begin{array}{l}\text { Na Ciência/Na Importância Política/Na Comer- } \\
\text { cialização/Na Formação/No Desenvolvimento } \\
\text { Econômico/No Meio Ambiente }\end{array}$ \\
\hline
\end{tabular}

Fonte: Adaptado de Ohayon (2007).

Em 2010, o Ministério do Planejamento, Orçamento e Gestão (MPOG) elaborou um guia metodológico para a construção de indicadores para programas do Governo Federal, com foco na estruturação de métodos, técnicas e instrumentos que possibilitassem a correta aferição dos resultados esperados.

Esse modelo classifica os indicadores, de acordo com a sua aplicação nas diferentes fases do ciclo de gestão, em: (a) insumo (input indicators); (b) processo (throughput indicators); (c) produto (output indicators), resultado (outcome indicators); e (d) impacto (impact indicators).

Para cada grupo de indicadores escolhidos, o modelo estabelece dois critérios para selecionar os tipos de indicadores, que são: critério eliminatório e critério classificatório. Uma vez selecionado o indicador, é feita uma análise de aderência de sua importância, conferindo-lhe um peso. Desse modo, constrói-se uma Matriz de Priorização de Indicadores que é de extrema relevância, pois essa explicita o ordenamento e a consequente classificação dos indicadores submetidos à avaliação de sua importância em relação a cada programa (BRASIL, MPOG, 2010, p. 30-63).

O modelo de Ohayon tem como vantagem a simplicidade, e por isso foi escolhido. "Sugere-se uma representação sistêmica simples, que tem a preocupação de ser operacional, bastante natural e clássica" (MoRAEs et al., 1989). 
Assim, o modelo procura destacar não apenas os tradicionais indicadores de inputs e outputs produzidos pelo Manual de Frascati (OCDE apud CNPq, 1978, p. 15), mas, sobretudo, os indicadores da dinâmica interna das atividades de CT\&l em nível institucional, assim como a utilização dos resultados (e não apenas esses) e os efeitos decorrentes. Cabe aqui ressaltar que não foi objeto do presente estudo analisar a qualidade dos indicadores da Fiocruz, ou mesmo as suas metas de desempenho, mas, sim, as dimensões que a Fiocruz usa para analisar suas atividades programáticas.

\section{Metodologia}

O estudo desenvolveu-se por métodos descritivos e exploratórios. É descritivo, pois, conforme Oliveira (2007, p. 68), "vai além do experimento: procura analisar fatos e/ou fenômenos, fazendo uma descrição detalhada de como se apresentam esses fatos e fenômenos ". Nesse caso, procura-se descrever o quadro atual de indicadores da Fiocruz, visando a classificá-los a partir do modelo conceitual.

A pesquisa documental restringiu-se à análise dos relatórios anuais de atividades e de gestão da Fiocruz (versão on-line), no período entre 2002 e 2011, pois são os dados mais atualizados de que se dispõe sobre a instituição.

A pesquisa bibliográfica objetivou buscar na literatura um modelo, a fim de confrontar os atuais indicadores utilizados pela instituição com os indicadores do modelo conceitual. O estudo também é exploratório, já que, a partir da coleta dos indicadores da instituição e da comparação com o modelo teórico, apontou as dimensões avaliativas não preenchidas pelos atuais indicadores e sugeriu novos indicadores.

Assim, primeiramente, foram identificados os principais sistemas de indicadores de CT\&I na literatura especializada, e foi realizada a escolha da instituição a ser pesquisada. Na segunda etapa, foi selecionada a documentação a ser analisada e realizada a comparação com o modelo conceitual proposto, conforme o Quadro 2.

A pesquisa se desenvolveu por estudo de caso, pois, segundo Yin (2005, p. 32), é um método de investigação de fenômeno contemporâneo dentro de seu contexto. A escolha da Fiocruz se deu por alguns motivos, entre eles, pelo fato de ser uma das maiores instituições de pesquisa do Brasil e de ter uma estrutura organizacional robusta. 
Quadro 2: Etapas da Pesquisa

\begin{tabular}{|l|l|l|l|}
\hline $\begin{array}{l}\text { Coleta de } \\
\text { informações }\end{array}$ & \multicolumn{1}{|c|}{$\begin{array}{c}\text { Tipo de } \\
\text { pesquisa }\end{array}$} & \multicolumn{1}{c|}{ Objetivos } & \multicolumn{1}{c|}{ Fonte } \\
\hline ETAPA 1 & $\begin{array}{l}\text { Pesquisa } \\
\text { bibliográfica } \\
\text { e } \\
\text { documental }\end{array}$ & $\begin{array}{l}\text { - Mapear principais } \\
\text { sistemas de indicadores } \\
\text { de CT\&I na literatura. }\end{array}$ & $\begin{array}{l}\text { Revistas especializadas; jornais científicos; } \\
\text { sites governamentais; artigos científicos. }\end{array}$ \\
\cline { 3 - 4 } & - Escolha da instituição. & $\begin{array}{l}\text { Entrevistas aos gestores, site das } \\
\text { instituições. }\end{array}$ \\
\hline ETAPA 2 & $\begin{array}{l}\text { Pesquisa de } \\
\text { campo }\end{array}$ & $\begin{array}{l}\text { - Selecionar e comparar } \\
\text { o modelo conceitual } \\
\text { com o usado pela Fiocruz. }\end{array}$ & $\begin{array}{l}\text { Relatórios de gestão; site da organização, } \\
\text { documentos internos. }\end{array}$ \\
\hline
\end{tabular}

Fonte: Elaborado pelos autores.

\section{Quadro de indicadores da Fundação Oswaldo Cruz (Fiocruz)}

A Fiocruz está vinculada ao Ministério da Saúde e foi criada em 25 de maio de 1900. Possui 16 unidades técnico-científicas e 4 unidades técnicoadministrativas, sendo a principal instituição de ciência e tecnologia no campo da saúde no País (Brasil, Ministério da Saúde, Fiocruz, 2012, p. 17). Os exemplos da participação da instituição na política de ciência e tecnologia em saúde, de medicamentos, de vacinas, de vigilância sanitária, propriedade industrial e de formação de recursos humanos para a área da saúde e na capacitação de técnicos para o SUS mostram a importância dessa instituição para a sociedade brasileira. Tem como missão

Produzir, disseminar e compartilhar conhecimentos e tecnologias voltados para o fortalecimento e a consolidação do SUS e que contribuam para a promoção da saúde e da qualidade de vida da população brasileira, a redução das desigualdades sociais e a dinâmica nacional de inovação, tendo a defesa do direito à saúde e da cidadania ampla como valores centrais (BRASIL, MINISTÉRIO da SaÚde, Fiocruz, 2012, p. 15).

O Relatório de Gestão da Fiocruz de 2011 possui uma abordagem informativa geral e programática que faz parte do Plano Plurianual (PPA) 2011 da instituição, cujos programas são apresentados na sua íntegra no Quadro 3. O relatório, que é constituído por 20 capítulos e 232 páginas, teve como foco para este estudo o seu item 2.3, intitulado "Programas de Governo sob Responsabilidade da Fiocruz", dentro do capítulo 2 - Planejamento e Gestão Orçamentária e Financeira -, que detalha os programas, ações, suas metas institucionais e os respectivos indicadores de desempenho. 
Quadro 3: Ações dos programas, os seus tipos e prioridades

\begin{tabular}{|c|c|c|c|c|}
\hline $\begin{array}{l}\text { Programa } \\
\text { № }\end{array}$ & $\begin{array}{l}\text { Ação } \\
\text { № }\end{array}$ & Nome da Ação & $\begin{array}{l}\text { Tipo de } \\
\text { Ação }\end{array}$ & $\begin{array}{l}\text { Priori- } \\
\text { dade }\end{array}$ \\
\hline 1201 & 7674 & Modernização das Unidades de Saúde da Fiocruz & $P$ & 3 \\
\hline 1201 & 8305 & $\begin{array}{l}\text { Atenção de Referência e Pesquisa Clínica em Patologias de } \\
\text { Alta Complexidade da Mulher, da Criança e do } \\
\text { Adolescente e em Doenças Infecciosas }\end{array}$ & A & 3 \\
\hline 1201 & 11PJ & Estruturação de Laboratórios de Pesquisas Biomédicas & $P$ & 3 \\
\hline 1201 & $20 A Q$ & $\begin{array}{l}\text { Coleções Biológicas e Outros Patrimônios da Ciência e da } \\
\text { Saúde no Brasil }\end{array}$ & A & 3 \\
\hline 1201 & 4363 & $\begin{array}{l}\text { Pesquisas e Inovações Tecnológicas em Tuberculose e } \\
\text { outras Pneumopatias no Centro de Referência Hélio Fraga }\end{array}$ & A & 3 \\
\hline 1201 & 6179 & $\begin{array}{l}\text { Comunicação e Informação para a Educação em Saúde e } \\
\text { em Ciência e Tecnologia em Saúde }\end{array}$ & A & 3 \\
\hline 1201 & $10 \mathrm{LF}$ & $\begin{array}{l}\text { Construção da Nova Sede Administrativa do Centro de } \\
\text { Pesquisas René Rachou em Belo Horizonte/MG }\end{array}$ & $\mathrm{P}$ & 1 \\
\hline 1201 & 2522 & Produção de Fármacos, Medicamentos e Fitoterápicos & $\mathrm{P}$ & 3 \\
\hline 1201 & 8315 & Pesquisa e Desenvolvimento Tecnológico em Saúde & $\mathrm{P}$ & 3 \\
\hline 1201 & $2 \mathrm{~b} 42$ & $\begin{array}{l}\text { Cooperação Técnica Nacional e Internacional em Ciência e } \\
\text { Tecnologia em Saúde }\end{array}$ & $\mathrm{P}$ & 3 \\
\hline 1201 & 20AJ & $\begin{array}{l}\text { Apoio a Pesquisas e Inovações Tecnológicas em Doenças } \\
\text { dos Trópicos (Ipepatro) }\end{array}$ & $\mathrm{P}$ & 4 \\
\hline 1201 & 7676 & $\begin{array}{l}\text { Construção do Centro de Desenvolvimento Tecnológico } \\
\text { em Saúde (CDTS) }\end{array}$ & $\mathrm{P}$ & 3 \\
\hline 1289 & 6174 & $\begin{array}{l}\text { Análise Técnico-Laboratorial da Qualidade de Produtos } \\
\text { Ofertados à População }\end{array}$ & A & 3 \\
\hline 1293 & 8415 & Manutenção e Funcionamento de Farmácias Populares & A & 1 \\
\hline 1436 & 8541 & $\begin{array}{l}\text { Educação Profissional, Educação Permanente e Pós- } \\
\text { Graduação em Saúde e em Ciência e Tecnologia em Saúde }\end{array}$ & A & 3 \\
\hline 1444 & 6031 & Imunobiológicos para Prevenção e Controle de Doenças & A & 3 \\
\hline 1444 & 6161 & $\begin{array}{l}\text { Aquisição, Acondicionamento e Distribuição de } \\
\text { Insumos/Prevenção e Controle de Doenças }\end{array}$ & A & 3 \\
\hline 1444 & 8327 & $\begin{array}{l}\text { Serviço Laboratorial de Referência para Controle de } \\
\text { Doenças }\end{array}$ & A & 3 \\
\hline 0750 & $20 \mathrm{CW}$ & Assistência Médica aos Servidores e Empregados & A & 3 \\
\hline 0750 & 2004 & $\begin{array}{l}\text { Assistência Médica e Odontológica aos Servidores, } \\
\text { Empregados e seus Dependentes }\end{array}$ & A & 4 \\
\hline 0750 & 2011 & $\begin{array}{l}\text { Auxílio-Transporte aos Servidores e Empregados Ação } \\
2011 \text { - Auxílio Transporte aos Servidores e Empregados }\end{array}$ & A & 4 \\
\hline 0750 & 2010 & $\begin{array}{l}\text { Assistência Pré-Escolar aos Dependentes dos Servidores e } \\
\text { Empregados }\end{array}$ & A & 4 \\
\hline 0750 & 2012 & Auxilio-Alimentação aos Servidores e Empregados & A & 4 \\
\hline
\end{tabular}

Legenda: $P=$ Projeto; $A=$ Atividade da Fiocruz.

Fonte: Adaptado pelos autores (Brasil, Ministério da Saúde, Fiocruz, 2012, p. 56-110).

O Quadro 3 mostra, nas colunas um e dois, como a Fiocruz atende e participa em cinco programas do Ministério da Saúde com ações de projetos e atividades ligadas à sua missão institucional, que são: Programa 1201 - CTICS - Ciência, Tecnologia e Inovação no Complexo da Saúde (8 projetos e 4 atividades); Programa 1444 - VPCDA - Vigilância, Prevenção e Controle de Doenças e Agravos 
(3 atividades); Programas 1289 - VPRDPCBS - Vigilância e Prevenção de Riscos Decorrentes da Produção e do Consumo de Bens e Serviços (1 atividade); Programa 1293 - AFIE - Assistência Farmacêutica e Insumos Estratégicos (1 atividade); e Programa 1436 - ATES - Aperfeiçoamento do Trabalho e da Educação na Saúde (1 atividade). Além desses, possui um programa próprio que é o Programa 0750 PAA - Programa de Apoio Administrativo (5 atividades).

A ampla variedade dos programas e ações do Plano Anual 2011 em que a Fiocruz atua mostra a abrangência e a diversidade de atividades desenvolvidas pela instituição. Os programas de Ciência, Tecnologia e Inovação em Saúde (1201) e de Educação Permanente e Qualificação Profissional (1436) são os mais abrangentes da instituição, uma vez que praticamente todas as unidades da Fiocruz desenvolvem algum projeto ou processo vinculado aos objetivos desses programas. Também tem grande relevância, para apoio do Sistema Único de Saúde (SUS) do Ministério da Saúde, a participação da Fiocruz nos programas de Assistência Farmacêutica (1293) e de Vigilância, Prevenção e Controle de Doenças e Agravos (1289).

No Quadro 4, estão descritas as finalidades das ações do Programa Ciência, Tecnologia e Inovação no Complexo da Saúde - 1201, em que esse programa busca a redução da dependência tecnológica brasileira em relação à produção de insumos estratégicos em saúde, ao aumento da capacidade de regulação do mercado e ao desenvolvimento de um sistema de inovação que articule de forma mais efetiva a pesquisa, o desenvolvimento tecnológico e a inovação em saúde. O alinhamento estratégico realizado em 2011 permitiu a destinação de recursos da ordem de $\mathrm{R} \$ 1$ bilhão, nos próximos quatro anos, para a criação de polos de desenvolvimento tecnológico e/ou de produção em biotecnologia nos Estados de Minas Gerais (polo de desenvolvimento tecnológico), Paraná (polo de desenvolvimento tecnológico), Ceará (plataforma vegetal para produção de imunobiológicos) e Rio de Janeiro (centro de processamento final de imunobiológicos - CPFI).

O Quadro 5 mostra as finalidades das ações dos demais programas, mais voltados às atividades relativas aos processos da Fiocruz. Cabe ressaltar que o Programa de Apoio Administrativo - 0750 está relacionado a ações da melhoria da qualidade de vida do servidor, com a finalidade da prevenção e controle de agravos à saúde, incluindo os agravos decorrentes do processo de trabalho.

As unidades da Fiocruz definem as metas de produção correspondentes a cada projeto/processo, registrando essas no Sistema Integrado de Informações Gerenciais (SIIG), sendo que cada projeto/processo está vinculado a um objetivo institucional que, por sua vez, está vinculado a uma ação do PPA (ver no Quadro 6). 


\section{Quadro 4: Finalidade das ações do Programa CTICS - 1201}

\begin{tabular}{|c|c|}
\hline Ação & Finalidade \\
\hline 7674 & $\begin{array}{l}\text { Modernizar as unidades da Fiocruz para permitir a implementação e a manutenção de sistemas } \\
\text { apropriados de gestão da qualidade, gestão ambiental, biossegurança e saúde do trabalhador. }\end{array}$ \\
\hline 8305 & $\begin{array}{l}\text { Realizar atenção de referência e pesquisa clínica nas áreas materno-infantil, de gestantes de alto } \\
\text { risco, crianças e adolescentes com patologia de alta complexidade e portadores de doenças } \\
\text { infecciosas. }\end{array}$ \\
\hline 11PJ & $\begin{array}{l}\text { Ampliar e modernizar as unidades da Fiocruz para possibilitar a incorporação de novas } \\
\text { atividades, assim como prover a infraestrutura necessária à gestão da qualidade, ambiental, } \\
\text { biossegurança e saúde do trabalhador. }\end{array}$ \\
\hline $20 A Q$ & $\begin{array}{l}\text { Identificar, preservar, valorizar e disponibilizar coleções biológicas e acervos museológicos, } \\
\text { arquivisticos e arquitetônicos da ciência e da saúde no Brasil. }\end{array}$ \\
\hline 4363 & $\begin{array}{l}\text { Promover a pesquisa e o desenvolvimento tecnológico em tuberculose e demais pneumopatias, } \\
\text { nas áreas das ciências biológicas aplicadas à saúde, das ciências humanas e sociais aplicadas à } \\
\text { saúde, da pesquisa clínica, da pesquisa epidemiológica e da avaliação de tecnologias em saúde, } \\
\text { desempenhando papel específico no Sistema Nacional de Vigilância em Saúde. }\end{array}$ \\
\hline 6179 & $\begin{array}{l}\text { Divulgar e difundir conhecimento científico e tecnológico em saúde para os profissionais de } \\
\text { saúde, pesquisadores e a população em geral. }\end{array}$ \\
\hline 10LF & Construção da nova sede administrativa do Centro de Pesquisas René Rachou. \\
\hline 2522 & $\begin{array}{l}\text { Suprir a demanda de medicamentos e fitoterápicos com a finalidade da utilização desses } \\
\text { produtos nos programas governamentais de assistência farmacêutica. }\end{array}$ \\
\hline 8315 & $\begin{array}{l}\text { Gerar conhecimento científico nas áreas das ciências biológicas aplicadas à saúde; das ciências } \\
\text { humanas e sociais aplicadas à saúde; da pesquisa clínica, da pesquisa epidemiológica e da } \\
\text { avaliação de tecnologias em saúde, a fim de assegurar a melhoria contínua das condições de } \\
\text { saúde da população e desenvolver novos fármacos, medicamentos, imunobiológicos e kits para } \\
\text { diagnóstico, e otimizar métodos e processos de saúde pública, visando à ampliação do acesso da } \\
\text { população às tecnologias de saúde e à melhoria dos serviços prestados pelo SUS. }\end{array}$ \\
\hline $2 \mathrm{~b} 42$ & $\begin{array}{l}\text { Apoiar técnica e cientificamente as unidades e redes dos sistemas nacionais de saúde, ciência e } \\
\text { tecnologia, educação e cultura, e ampliar a atuação internacional do Brasil no âmbito da saúde, } \\
\text { especialmente em relação aos países africanos de língua portuguesa e aos países da América } \\
\text { Latina. }\end{array}$ \\
\hline 20AJ & $\begin{array}{l}\text { Promover a pesquisa e o desenvolvimento tecnológico nas áreas das ciências biológicas aplicadas } \\
\text { à saúde; das ciências humanas e sociais aplicadas à saúde; da pesquisa clínica; da pesquisa } \\
\text { epidemiológica; e da avaliação de tecnologias em saúde, com ênfase nas patologias tropicais, a } \\
\text { fim de assegurar a melhoria das condições de saúde da população, em especial dos povos } \\
\text { amazônicos. }\end{array}$ \\
\hline 7676 & $\begin{array}{l}\text { Disponibilização de infraestrutura e competência profissional necessária para transformação de } \\
\text { ideias, processos e produtos em bens de saúde, que estejam prontos para processamento } \\
\text { industrial. Mecanismo para ampliar a oferta de produtos/insumos de saúde à população. }\end{array}$ \\
\hline
\end{tabular}

Fonte: Adaptado pelos autores (Brasil, Ministério da Saúde, Fiocruz, p. 56-110, 2012).

Essas metas são sistematizadas e consolidadas, passando a compor o quadro de metas físicas das ações/programas do PPA-Fiocruz (Quadro 6). O grau de realização das metas programadas para cada ação/programa do PPA-Fiocruz é a base da avaliação dos resultados institucionais, medidos mediante os indicadores de eficácia. Em todas as análises de execução do plano anual, destacam-se os produtos-índice de cada ação, compreendidos como aqueles que melhor representam o objetivo final de cada ação e que são informados aos Ministérios da Saúde e do Planejamento por meio do PlamSUS e do SigPlan respectivamente. 


\section{Quadro 5: Finalidades das ações dos programas VPCDA (1444); VPRDPCBS (1289); Afie (1293); Ates (1436) e PAA (0750)}

\begin{tabular}{|c|c|c|}
\hline $\begin{array}{l}\text { Pro- } \\
\text { grama }\end{array}$ & $\begin{array}{l}\text { Ação } \\
\text { № }\end{array}$ & Finalidade \\
\hline 1289 & 6174 & $\begin{array}{l}\text { Assegurar a prevenção da ocorrência de possiveis efeitos indesejáveis à saúde } \\
\text { humana decorrentes da utilização de insumos, produtos ou serviços inadequados } \\
\text { e sujeitos à vigilância sanitária. }\end{array}$ \\
\hline 1293 & 8145 & $\begin{array}{l}\text { Assegurar a manutenção e funcionamento do Programa Farmácia Popular em } \\
\text { unidades exclusivas do programa, bem como na rede privada de farmácias } \\
\text { credenciadas pelo Ministério da Saúde. }\end{array}$ \\
\hline 1436 & 8541 & $\begin{array}{l}\text { Ampliar a qualificação dos trabalhadores do SUS e do sistema nacional de ciência } \\
\text { e tecnologia em saúde, mediante atividades de ensino em niveis de pós-graduação } \\
\text { stricto e lato sensu e de formação profissional em saúde. }\end{array}$ \\
\hline \multirow[t]{3}{*}{1444} & 6031 & $\begin{array}{l}\text { Suprir a rede pública de serviços de saúde com imunobiológicos na quantidade } \\
\text { necessária, de forma a reduzir o indice de mortalidade por doenças imunopreve- } \\
\text { niveis e otimizar resultados com relação ao controle e erradicação de doenças. }\end{array}$ \\
\hline & 6161 & $\begin{array}{l}\text { Disponibilizar oportunamente os insumos estratégicos necessários para a adoção } \\
\text { de medidas de prevenção e controle de doenças em tempo oportuno, com vistas } \\
\text { a reduzir a morbimortalidade decorrente das doenças e agravos prevalentes. }\end{array}$ \\
\hline & 8327 & $\begin{array}{l}\text { Prestar serviços de referência no contexto do Sistema Nacional de Laboratórios } \\
\text { de Saúde Pública, realizando análises de confirmação diagnóstica de doenças } \\
\text { prevalentes, emergentes e reemergentes. Ampliar a capacidade nacional de } \\
\text { vigilância em saúde, por meio da produção de conhecimentos, metodologias e } \\
\text { modelos de intervenção e mediante parcerias nacionais e internacionais. }\end{array}$ \\
\hline \multirow[t]{5}{*}{0750} & $20 \mathrm{CW}$ & $\begin{array}{l}\text { Realizar exames de saúde periódicos a servidores e empregados da Fiocruz, como } \\
\text { medida de prevenção e controle de agravos à saúde, incluindo os agravos } \\
\text { decorrentes do processo de trabalho. }\end{array}$ \\
\hline & 2004 & $\begin{array}{l}\text { Proporcionar aos servidores, empregados, seus dependentes e pensionistas } \\
\text { condições para manutenção da saúde física e mental. }\end{array}$ \\
\hline & 2011 & $\begin{array}{l}\text { Custeio parcial das despesas realizadas com transporte coletivo municipal, } \\
\text { intermunicipal ou interestadual pelos servidores e empregados públicos da } \\
\text { administração federal direta, autárquica e fundacional da União, nos } \\
\text { deslocamentos de suas residências para os locais de trabalho e vice-versa, } \\
\text { de acordo com a Lei no } 7.418 / 85 \text { e alterações, e Medida Provisória no } 2.165-36 \text {, } \\
\text { de } 23 / 08 / 2001 \text {. }\end{array}$ \\
\hline & 2010 & $\begin{array}{l}\text { Oferecer aos servidores, durante a jornada de trabalho, condições adequadas de } \\
\text { atendimento aos seus dependentes, conforme art. } 3 \circ \text { do Decreto } n \circ 977 \text {, de } \\
10 / 11 / 93 \text {. }\end{array}$ \\
\hline & 2012 & $\begin{array}{l}\text { Conceder o auxílio-alimentação, pago na proporção dos dias trabalhados e } \\
\text { custeado com recursos do órgão ou entidade de lotação ou exercício do servidor } \\
\text { ou empregado, aquisição de vale ou ticket-alimentação ou refeição, ou } \\
\text { manutenção de refeitório. }\end{array}$ \\
\hline
\end{tabular}

Fonte: Adaptado pelos autores (BrasiL, Ministério da Saúde, FIocruz, 2012, p. 56-110).

A análise global do desempenho é feita com base nos produtos-índice. A meta revisada representa o compromisso efetivamente assumido pela Fiocruz a partir da disponibilidade real de recursos alocados, após a aprovação final da lei 
orçamentária anual (LOA). As metas revisadas são utilizadas para complementar a análise do grau de realização das metas estabelecidas e para estabelecer comparações entre unidades, ações e objetivos do Plano Anual da Fiocruz. O Quadro 6 mostra um panorama da execução física das ações sob responsabilidade da Fiocruz.

\section{Quadro 6: Principais indicadores da Fiocruz}

\begin{tabular}{|c|c|c|c|c|}
\hline Ação & Indicador & $\begin{array}{l}\text { Meta } \\
\text { Prevista } \\
\text { no PPA } \\
2011\end{array}$ & $\begin{array}{l}\text { Meta } \\
\text { Revista } \\
2011\end{array}$ & $\begin{array}{l}\text { Meta } \\
\text { Realizada } \\
2011\end{array}$ \\
\hline 7674 & № de unidades modernizadas & 28 & $26(\downarrow)$ & $26(\downarrow)$ \\
\hline 8305 & № de pacientes atendidos & 75.000 & $76.348(\uparrow)$ & $78.400(\uparrow)$ \\
\hline $11 \mathrm{PJ}$ & Percentagem de obras executadas & 23 & $57,9(\uparrow)$ & 64( $\uparrow)$ \\
\hline $20 A Q$ & № de coleções biológicas mantidas & 29 & $29(\sim)$ & $27(\downarrow)$ \\
\hline 4363 & $\begin{array}{l}\text { № de pesquisas realizadas no Centro de Referência } \\
\text { Hélio Fraga* }\end{array}$ & 13 & $19(\uparrow)$ & $16(\uparrow)$ \\
\hline 6179 & $\begin{array}{l}\text { Material produzido de comunicação e informação } \\
\text { para a educação em saúde e ciência e tecnologia em } \\
\text { saúde }\end{array}$ & 1.500 .000 & $\begin{array}{l}1.590 .320 \\
(\uparrow)\end{array}$ & $\begin{array}{l}1.249 .630 \\
(\downarrow)\end{array}$ \\
\hline $10 \mathrm{LF}$ & № de sedes construídas & 5 & $0(\downarrow)$ & $0(\downarrow)$ \\
\hline 2522 & № de unidades farmacêuticas produzidas & 2.500 .000 & $509.270(\downarrow)$ & $494.481(\downarrow)$ \\
\hline 8315 & № de pesquisas realizadas na Fiocruz & 1.700 & $1.685(\downarrow)$ & $1.427(\downarrow)$ \\
\hline $2 b 42$ & № de cooperações técnicas mantidas & 600 & $600(\sim)$ & $526(\downarrow)$ \\
\hline 20AJ & № de pesquisas apoiadas & 35 & $62(\uparrow)$ & 60( $\uparrow)$ \\
\hline 7676 & Percentagem do novo centro construído & 27 & $46,2(\uparrow)$ & $65(\uparrow)$ \\
\hline 6174 & № de laudos emitidos & 4.500 & $4.020(\downarrow)$ & $5.229(\uparrow)$ \\
\hline 8415 & № de farmácias populareses & 136 & $550(\uparrow)$ & $554(\uparrow)$ \\
\hline 8541 & № de alunos egressos & 13.590 & $11.715(\downarrow)$ & $12.197(\downarrow)$ \\
\hline 6031 & № de doses de vacinas & 192.150 & $\begin{array}{l}165.639 \\
(\downarrow)\end{array}$ & $\begin{array}{l}140.924 \\
(\downarrow)\end{array}$ \\
\hline 6161 & № de reagentes e insumos para diagnóstico $\left(\mathrm{em} 10^{3}\right)$ & 5.700 & $7.947(\uparrow)$ & $5.919(\uparrow)$ \\
\hline 8327 & № de exames de laboratório realizados & 250.000 & $\begin{array}{l}155.899 \\
(\downarrow)\end{array}$ & $\begin{array}{l}317.652 \\
(\uparrow)\end{array}$ \\
\hline $20 \mathrm{CW}$ & № de exames periódicos dos servidores & 3.273 & $3.273(\sim)$ & $152(\downarrow)$ \\
\hline 2004 & $\begin{array}{l}\text { № de atendimento de servidores (saúde e } \\
\text { odontológico) }\end{array}$ & 10.435 & $10.435(\sim)$ & $14.206(\uparrow)$ \\
\hline 2011 & № de servidores beneficiados com auxilio-transporte & 1.748 & $1.748(\sim)$ & $1.320(\downarrow)$ \\
\hline 2010 & № de crianças assistidas (assistência pré-escolar) & 546 & $546(\sim)$ & $352(\downarrow)$ \\
\hline 2012 & $\begin{array}{l}\text { № de servidores beneficiados com a auxílio- } \\
\text { alimentação }\end{array}$ & 6.643 & $6.643(\sim)$ & $4.399(\downarrow)$ \\
\hline
\end{tabular}

Fonte: Adaptado pelos autores (Brasil, Ministério dA SAÚde, Fiocruz, 2012, p. 56).

Legenda: $\mathrm{P}=$ Projeto; $\mathrm{A}=$ Atividade da Fiocruz. ${ }^{*} \mathrm{O}$ Centro de Referência Hélio Fraga é uma das unidades da Fiocruz. $(\uparrow)=$ Maior que PPA, $(\downarrow)=$ Menor que PPA, $(\sim)=$ semelhante ao PPA. 
Os indicadores apresentados pela Fiocruz, conforme o Quadro 6, não apresentam uma correlação entre si, dificultando o processo de avaliação. Verifica-se que, das 23 metas previstas no PPA 2011, apenas oito foram revistas para baixo (ver, no Quadro 6, setas para baixo), sendo que sete não foram mexidas, significando que as metas foram bem estabelecidas originalmente com as unidades. Observa-se que a maioria das metas realizadas, perfazendo um total de 13 , foram um pouco menores que 0 proposto inicialmente pelo PPA 2011, e que 10 foram maiores do que o planejado, em princípio demonstrando o sucesso da instituição quanto ao alcance de suas metas.

No entanto, discute-se a capacidade da ferramenta em traduzir a realidade, supondo que está relacionada com a diversidade dos aspectos avaliados. Para o processo de análise crítica de desempenho institucional, é preciso que os indicadores estejam agrupados em conjuntos homogêneos de dimensões e subdimensões, conforme o modelo conceitual preconizado no Quadro 1, a fim de observar lacunas, buscando aperfeiçoar o modelo de avaliação da instituição.

Verifica-se que o sistema de metas adotado pela Fiocruz não possui uma metodologia avaliativa capaz de privilegiar mais os indicadores estratégicos do que os demais, e isso pode dificultar uma análise mais pertinente relacionada aos aspectos administrativo-financeiros e de recursos humanos.

\section{Análise comparativa entre os atuais indicadores da Fiocruz e o modelo conceitual}

A partir da comparação entre o modelo conceitual e o observado, conforme Quadro 7, constata-se a ausência de avaliação dos efeitos e impactos das atividades da Fiocruz no desenvolvimento científico-tecnológico. Supõe-se que o modelo de avaliação utilizado seja baseado num sistema fechado, não interagindo com o ambiente externo.

Constata-se ainda a maior presença de indicadores de resultados diretos e indiretos. Como resultado direto, pode-se apontar notavelmente o número de laudos ou exames. Essas atividades podem ser analisadas pelos resultados diretos que nem sempre são indicadores típicos de CT\&l, como exposto anteriormente, e também pelos resultados de atividade de produção científica e técnica (outputs indiretos). Esses são compostos pelos índices de publicação, de livros editados e os indicadores já citados de pesquisa. As seguintes observações podem ser extraídas do Quadro 7:

- A existência de apenas quatro indicadores relacionados à subdimensão de recursos humanos, referindo-se ao levantamento de número de servidores beneficiados. 
- Há apenas um indicador de recursos financeiros, e faltam indicadores para mensurar os recursos informacionais e organizacionais.

- A presença de apenas um indicador de gestão que mede o número de atendimentos pela Ouvidoria, bem como a subdimensão de cooperação e de abertura possui um indicador apenas.

- Não existem indicadores específicos relativos ao planejamento estratégico. Observa-se que as ações programáticas, em sua constituição, estão alinhadas com a missão, a visão e os valores que compõem as diretrizes da instituição. No entanto, não se observa, para que seja possível uma análise aprofundada do desempenho das metas, a presença dos fatores críticos de sucesso ${ }^{1}$.

- Para a dimensão "resultados diretos", são encontrados indicadores para todas as subdimensões de pesquisas.

- Não são encontrados indicadores para avaliação da utilização dos resultados e dos seus efeitos em nenhuma dimensão apontada.

Pode-se afirmar, portanto, que o modelo utilizado pela Fiocruz é bastante limitado, frente ao proposto por Ohayon, já que faltam indicadores informacionais, organizacionais e de utilização e efeitos dos resultados. A importância dos primeiros está no fato de mapear os recursos informacionais que estão à disposição na instituição. Não adianta ter orçamento ou uma equipe de trabalho, se não houver investimento em boas práticas organizacionais que aumentem a eficiência dos processos e permitam à equipe exercer todo o seu potencial. A informação, matéria-prima das atividades de CT\&I, deve ser considerada, pois indica o grau de acessibilidade da equipe ao sistema de CT\&I, dando-lhes subsídios para novas pesquisas.

O Quadro 7 mostra que algumas subdimensões têm uma quantidade maior de indicadores e outras, poucos ou nenhum, bem como alguns não possuem metas definidas. Assim, por exemplo, a falta de indicadores relacionados à mensuração dos resultados das atividades na orientação de teses faz com que a Fiocruz não tenha como monitorar a qualidade dos seus resultados enquanto instituição de ensino. Sem essas informações, não é possível posicionar o que é feito efetivamente pela instituição em termos de formação de pesquisadores com o papel estratégico exercido na formação de cientistas para o País.

\footnotetext{
${ }^{1}$ Ressalvando-se que, para os documentos analisados (Relatório Anual de Gestão da Fiocruz 2011), não foram encontradas menções a esses fatores, nem seus indicadores.
} 


\section{Quadro 7: Dimensões e subdimensões de indicadores na Fiocruz}

\begin{tabular}{|c|c|c|c|c|}
\hline Dimensões & Subdimensões & Indicadores da Fiocruz & Ação & Meta \\
\hline \multirow{11}{*}{$\begin{array}{l}\text { Recursos } \\
\text { (inputs) }\end{array}$} & \multirow[t]{5}{*}{ - Humanos } & № de exames periódicos dos servidores & $20 \mathrm{CW}$ & $\operatorname{sim}$ \\
\hline & & № de atendimento de servidores (saúde e odontológico) & 2004 & $\operatorname{sim}$ \\
\hline & & № de servidores beneficiados com auxilio-transporte & 2011 & $\operatorname{sim}$ \\
\hline & & № de crianças assistidas (assistência pré-escolar) & 2010 & sim \\
\hline & & № de servidores beneficiados com auxilio-alimentação & 2012 & $\operatorname{sim}$ \\
\hline & - Financeiros & Custo médio por unidade farmacêutica produzida & 2522 & não \\
\hline & \multirow{3}{*}{$\begin{array}{l}\text { - Materiais e Espaço } \\
\text { Físico }\end{array}$} & № de unidades modernizadas & 7674 & sim \\
\hline & & Percentagem de obras executadas & $11 \mathrm{PJ}$ & $\operatorname{sim}$ \\
\hline & & Percentagem do novo centro construído & 7676 & sim \\
\hline & - Informacionais & Não tem & $\ldots$ & $\ldots$ \\
\hline & - Organizacionais & Não tem & $\cdots$ & $\ldots$ \\
\hline \multirow{3}{*}{$\begin{array}{l}\text { Dinâmica das } \\
\text { atividades de } \\
\text { CT\&l } \\
\text { (Processo) }\end{array}$} & - Gestão & Número de manifestações atendidas em Ouvidoria & 7674 & não \\
\hline & $\begin{array}{l}\text { - Cooperação e } \\
\text { Abertura }\end{array}$ & $\begin{array}{l}\text { Número de cooperações técnicas nacionais einternacionais } \\
\text { em ciência e tecnologia em saúde }\end{array}$ & $2 \mathrm{~b} 42$ & $\operatorname{sim}$ \\
\hline & - Estratégia & Não tem & $\ldots$ & ....... \\
\hline \multirow{34}{*}{$\begin{array}{l}\text { Resultados } \\
\text { Diretos } \\
\text { (Outputs } \\
\text { Diretos) }\end{array}$} & \multirow{21}{*}{ - Produção } & Patrimônio arquitetônico preservado & $20 A Q$ & não \\
\hline & & № total de pacientes atendidos pelos hospitais & 8305 & $\operatorname{sim}$ \\
\hline & & № de consultas ambulatoriais & 8305 & $\operatorname{sim}$ \\
\hline & & № de internações & 8305 & $\operatorname{sim}$ \\
\hline & & $\mathrm{N}^{\circ}$ de exames realizados nos hospitais & 8305 & $\operatorname{sim}$ \\
\hline & & $\mathrm{N} \cong$ de atendimentos domiciliares realizados & 8305 & $\operatorname{sim}$ \\
\hline & & № de cirurgias & 8305 & $\operatorname{sim}$ \\
\hline & & № de consultas & 8305 & $\operatorname{sim}$ \\
\hline & & Taxas de infecção hospitalar nas unidades assistenciais & 8305 & não \\
\hline & & Taxas de mortalidade hospitalar nas unidades assistenciais & 8305 & não \\
\hline & & Tempo médio de permanência nas unidades assistenciais & 8305 & não \\
\hline & & Taxa de ocupação hospitalar nas unidades assistenciais & 8305 & não \\
\hline & & № de unidades farmacêuticas produzidas & 2522 & $\operatorname{sim}$ \\
\hline & & № de laudos emitidos & 6174 & $\operatorname{sim}$ \\
\hline & & № de usuários atendidos pelo Programa Farmácia Popular & 8415 & não \\
\hline & & № de farmácias populares & 8415 & $\operatorname{sim}$ \\
\hline & & $\begin{array}{l}\text { № de unidades farmacêuticas dispensadas, Programa } \\
\text { Farmácia Popular do Brasil }\end{array}$ & 8415 & não \\
\hline & & № de doses de vacinas & 6031 & $\operatorname{sim}$ \\
\hline & & № de reagentes e insumos para diagnóstico (em $10^{3}$ ) & 6161 & $\operatorname{sim}$ \\
\hline & & № de exames laboratoriais realizados & 8327 & $\operatorname{sim}$ \\
\hline & & $\begin{array}{l}\text { Percentual de exames laboratoriais de referência realizados } \\
\text { pelas unidades }\end{array}$ & 8327 & não \\
\hline & \multirow{5}{*}{ - Pesquisas } & Coleções biológicas mantidas & $20 A Q$ & sim \\
\hline & & № de acervos arquivísticos preservados & 20AQ & $\operatorname{sim}$ \\
\hline & & № de acervos museológicos preservados & 20AQ & $\operatorname{sim}$ \\
\hline & & $\begin{array}{l}\text { Pesquisas realizadas no Centro de Referência Hélio Fraga da } \\
\text { Fiocruz }\end{array}$ & 4363 & $\operatorname{sim}$ \\
\hline & & № de pesquisas apoiadas & 20AJ & $\operatorname{sim}$ \\
\hline & \multirow{3}{*}{ - Educação } & № de alunos egressos & 8541 & sim \\
\hline & & Percentagem de alunos egressos por unidade (Fiocruz) & 8541 & $\operatorname{sim}$ \\
\hline & & Número de alunos egressos por tipo de curso (Fiocruz) & 8541 & não \\
\hline & \multirow{5}{*}{ - Difusão } & № de usuários de mídia virtual & 6179 & $\operatorname{sim}$ \\
\hline & & № de usuários de bibliotecas & 6179 & $\operatorname{sim}$ \\
\hline & & № de usuário atendidos em exposições do Museu da Vida & 6179 & $\operatorname{sim}$ \\
\hline & & NN2 de eventos técnico-científicos realizados & 6179 & não \\
\hline & & № de apresentaçōes em eventos científicos & 8315 & sim \\
\hline
\end{tabular}




\begin{tabular}{|c|c|c|c|c|}
\hline Dimensões & Subdimensões & Indicadores da Fiocruz & Ação & Meta \\
\hline \multirow{15}{*}{$\begin{array}{l}\text { Atividade, } \\
\text { Produção } \\
\text { Cientifica e } \\
\text { Técnica } \\
\text { (outputs } \\
\text { indiretos) }\end{array}$} & \multirow{2}{*}{$\begin{array}{l}\text { - Projetos, } \\
\text { Programas e Ações } \\
\text { Desenvolvidas }\end{array}$} & № de pesquisas realizadas na Fiocruz & 8315 & Sim \\
\hline & & $\begin{array}{l}\text { № de tecnologias trans/incorporadas pela Fiocruz ao } \\
\text { setor produtivo }\end{array}$ & 8315 & $\operatorname{sim}$ \\
\hline & \multirow{12}{*}{$\begin{array}{l}\text { - Publicações de } \\
\text { Artigos e Teses }\end{array}$} & $\begin{array}{l}\text { Material produzido de comunicação e informação para a } \\
\text { educação em saúde e em ciência e tecnologia em saúde }\end{array}$ & 6179 & $\operatorname{sim}$ \\
\hline & & № de artigos publicados pelas revistas cientificas da Fiocruz & 6179 & $\operatorname{sim}$ \\
\hline & & № de citações dos periódicos editados pela Fiocruz & 6179 & não \\
\hline & & № de publicação em revista indexada & 8315 & $\operatorname{sim}$ \\
\hline & & № puublicações em revistas não indexadas & 8315 & $\operatorname{sim}$ \\
\hline & & № de publicações em capítulos de livros & 8315 & sim \\
\hline & & № de livros publicados & 8315 & sim \\
\hline & & № de pedidos de patente requeridos no Brasil & 8315 & $\operatorname{sim}$ \\
\hline & & № de pedidos de patente requeridos no exterior & 8315 & $\operatorname{sim}$ \\
\hline & & $\begin{array}{l}\text { Percentual das publicações em revistas indexadas, segundo } \\
\text { unidades da Fiocruz }\end{array}$ & 8315 & $\operatorname{sim}$ \\
\hline & & Produção científica por pesquisador/doutor e por doutor & 8315 & não \\
\hline & & Índice de publicações em revistas indexadas & 8315 & não \\
\hline & $\begin{array}{l}\text { - Orientação de } \\
\text { Teses }\end{array}$ & Não tem & $\ldots$ & $\ldots$ \\
\hline \multirow{3}{*}{$\begin{array}{l}\text { Utilização dos } \\
\text { Resultados }\end{array}$} & - Bibliométricos & $\begin{array}{l}\text { Fator de impacto em período de três anos dos periodicos } \\
\text { científicos da Fiocruz }\end{array}$ & 6179 & não \\
\hline & - Físicos & \multirow{8}{*}{ Não encontrados } & $\ldots . . .$. & $\ldots$ \\
\hline & - Comercialização & & $\ldots$ & $\ldots$ \\
\hline \multirow{6}{*}{ Efeitos } & - Na Ciência & & …... & ....... \\
\hline & $\begin{array}{l}\text { - Na Importância } \\
\text { Política }\end{array}$ & & $\cdots$ & $\cdots$ \\
\hline & - Na Comercialização & & $\ldots . . .$. & $\ldots . . .$. \\
\hline & - Na Formação & & $\ldots . .$. & ....... \\
\hline & $\begin{array}{l}\text { - No Desenvolvimento } \\
\text { Econômico }\end{array}$ & & $\ldots$ & $\ldots$ \\
\hline & - Meio Ambiente & & $\ldots . . .1$ & $\ldots . . . .$. \\
\hline
\end{tabular}

Fonte: Adaptado de Ohayon (2007).

Quanto à ausência dos indicadores de utilização de resultados e de efeitos, não permite mensurar a importância desses resultados para a instituição, ou seja, questões relevantes ficam sem respostas, a exemplo: "os resultados das atividades da Fiocruz são relevantes para a comunidade internacional?", "essas geram bom retorno financeiro?" ou "quanto dos resultados alcançados geram tecnologia de ponta para o desenvolvimento tecnológico do País?". A insuficiência do quadro de indicadores não está somente na ausência de alguns deles, mas também na característica limitada dos indicadores presentes.

Observando os indicadores de recursos humanos, não há como saber o tamanho da equipe de pesquisa, nem dos funcionários de apoio. Como os recursos humanos não fazem parte de nenhum programa relacionado ao PPA2011 do MS, logo não existe a preocupação da instituição em relacionar e monitorar esse tipo de subdimensão. Assim, não há uma relação entre as ações estabelecidas e o quadro de pessoal existente. Nem mesmo é demonstrada a faixa etária do quadro de pesquisadores, o que poderia responder a questões do tipo: se a Fiocruz possui um quadro experiente em termos de tempo de serviço e parte do quadro está prestes a se aposentar, o que indicaria a 
necessidade de novas contratações, ou se ocorre o oposto, o quadro é jovem demais, indicando necessidade de treinamento.

Quanto aos recursos financeiros, o modelo atual não informa, por exemplo, o quanto é aplicado em pesquisas por área temática. Essa informação é importante para se determinar qual a política de desenvolvimento de pesquisa da instituição, se todas as linhas de pesquisa têm tratamento equânime, ou se há prioridades entre as áreas de pesquisa. Também não estão discriminados os recursos financeiros aplicados às demais atividades-fim da Fiocruz, como a pós-graduação. Enfim, sem o mapeamento por completo dos recursos que estão à disposição da organização, torna-se menos eficiente o processo de transformação desses recursos nos resultados desejados. No que se refere aos indicadores de gestão, não há indicadores relacionados com o processo de execução orçamentária. Informações quanto ao uso efetivo dos recursos empregados, por exemplo, não são consideradas. Isso é importante para se determinar a real produtividade frente aos recursos empregados.

No que diz respeito aos indicadores de utilização dos resultados, faltam aqueles para mensurar as atividades de comercialização como, por exemplo, a venda de patentes ou o recebimento de royalties. Também, pode-se incluir a subdimensão da utilização dos espaços físicos para a obtenção de receitas complementares.

Sobre indicadores estratégicos, é importante ressaltar a ausência de mensuração dos fatores críticos de sucesso (FCS). Para Rockart (1979 apud MülLER, 2003, p. 37), FCS são áreas essenciais para o sucesso na obtenção das metas e, portanto, a execução das atividades relacionadas deve ser traduzida em indicadores que monitorem seus desempenhos. Os FCS seriam os eventos interventores na obtenção das metas, isto é, condições que devem ser satisfeitas.

\section{Proposta de indicadores complementares para a Fiocruz}

O Quadro 8 preenche as lacunas a partir do fornecimento de alguns exemplos de indicadores oriundos da literatura especializada, e algumas proposições do presente estudo que poderiam ser acrescentadas e, consequentemente, melhor avaliar as atividades de CT\&l da instituição.

Dos 29 indicadores sugeridos no Quadro 8, pode-se afirmar que todos são relativamente fáceis de serem medidos, fazendo com que o custo da medição seja negligenciável frente aos benefícios que podem trazer para a tomada de decisão dos gestores da Fiocruz. 
Quadro 8: Proposta de indicadores para a Fiocruz segundo o modelo proposto

\begin{tabular}{|c|c|c|}
\hline Dimensões & Subdimensões & Proposta de indicadores \\
\hline \multirow{10}{*}{ Recursos (inputs) } & \multirow[t]{2}{*}{-Recursos Humanos } & Índice de qualificação do corpo de pesquisadores por título acadêmico \\
\hline & & Índice de utilização de recursos humanos de pesquisadores \\
\hline & \multirow[t]{2}{*}{-Recursos Financeiros } & Total aplicado em pesquisa por área de pesquisa \\
\hline & & Total aplicado em pesquisa por pesquisador \\
\hline & \multirow[t]{2}{*}{$\begin{array}{l}\text {-Recursos Materiais e } \\
\text { Espaço Fisico }\end{array}$} & $\begin{array}{l}\text { Índice de disponibilidade de equipamentos científicos por área de } \\
\text { pesquisa }\end{array}$ \\
\hline & & Índice de disponibilidade de laboratórios \\
\hline & \multirow{2}{*}{$\begin{array}{l}\text {-Recursos } \\
\text { Informacionais }\end{array}$} & Índice de produção de conhecimentos \\
\hline & & Acervo cadastrado na base digital \\
\hline & \multirow{2}{*}{$\begin{array}{l}\text {-Recursos } \\
\text { Organizacionais }\end{array}$} & Número de linhas de pesquisa por grupo temático \\
\hline & & Índice de produtividade do pesquisador \\
\hline \multirow{2}{*}{$\begin{array}{l}\text { Dinâmica das } \\
\text { atividades de } \\
\text { CT\&I (processo) }\end{array}$} & \multirow[b]{2}{*}{ - Gestão } & Tempo de experiência na instituição de pesquisa (por área) \\
\hline & & Grau de projetos abandonados \\
\hline \multirow{4}{*}{$\begin{array}{l}\text { Resultados } \\
\text { Diretos ( outputs } \\
\text { diretos) }\end{array}$} & \multirow{2}{*}{ - Educação } & Índice de eficiência do mestrado \\
\hline & & Índice de eficiência do doutorado \\
\hline & \multirow[t]{2}{*}{ - Difusão } & Acréscimo do número de alunos na vocação científica \\
\hline & & Acréscimo do número de projetos desenvolvidos \\
\hline \multirow{7}{*}{$\begin{array}{l}\text { Utilização dos } \\
\text { Resultados }\end{array}$} & - Físicos & Projetos implantados/comercializados/patentes depositadas \\
\hline & - Bibliométricos & Qualidade da pesquisa \\
\hline & \multirow[t]{5}{*}{ - Para Comercialização } & Novos contratos firmados \\
\hline & & Duração média dos contratos \\
\hline & & Valor médio dos contratos \\
\hline & & Receita média gerada pela venda de equipam entos \\
\hline & & Receita gerada pelos cursos, seminários e demais eventos científicos \\
\hline \multirow{6}{*}{ Efeitos } & - Na Ciência & $\begin{array}{l}\text { Aumento no número de artigos e publicações científicas em Física no } \\
\text { Brasil }\end{array}$ \\
\hline & $\begin{array}{l}\text { - Na Importância } \\
\text { Politica }\end{array}$ & Aumento do orçamento para atividades de CT\&I (em Saúde no Brasil) \\
\hline & - Na Comercialização & $\begin{array}{l}\text { Aumento na demanda por pesquisas pelas empresas (em Biologia no } \\
\text { Brasil) }\end{array}$ \\
\hline & $\begin{array}{l}\text {-No Desenvolvimento } \\
\text { Econômico }\end{array}$ & Receita média do conhecimento \\
\hline & - Na Formação & Aumento na demanda por cursos de pós-graduação em Saúde - Brasil \\
\hline & - No Meio Ambiente & Impacto ambiental \\
\hline
\end{tabular}

Fonte: Elaborado pelos autores.

\section{Considerações finais}

A avaliação é uma das importantes ferramentas para que a gestão da ciência, tecnologia e inovação seja eficiente e eficaz. Para que seu uso seja eficaz, a avaliação deve estar apoiada basicamente em indicadores verificáveis e confiáveis, e que permitam comparações de desempenho.

Ficou evidenciado que, apesar de a Fiocruz não adotar nenhum modelo de avaliação explícito para a seleção e análise do seu sistema de indicadores programáticos, essa possui de forma estruturada uma gama de indicadores por ações para cada programa institucional. Assim, verificou-se nessa pesquisa que os 
indicadores de CT\&I utilizados pela Fiocruz são pertinentes e permitem comparações com aqueles de outras instituições nacionais e internacionais. Ressalta-se que o quadro de indicadores utilizado pela Fiocruz apresenta indicadores de recursos humanos deficientes e indicadores de resultados insuficientes para uma avaliação mais completa das atividades de CT\&I. O quadro de indicadores utilizado não exprime, de fato, as condições do ambiente no qual a instituição está inserida (condições sociais, econômicas, ambientais e tecnológicas).

Verificou-se, também, que, mais do que interação, faltou ao quadro de indicadores utilizado pela Fiocruz o conceito de sistema aberto, ou seja, a mensuração necessária dos impactos gerados no ambiente pelas atividades de CT\&। desenvolvidas pela instituição.

Portanto, pode-se concluir que o conjunto de indicadores utilizado pela Fiocruz é limitado ao conceito de sistema fechado, isto é, não considera a interação com o ambiente externo. Embora possa ser apontado um indicador de cooperação, esse foi desenvolvido como um indicador de resultado das atividades de CT\&I da Fiocruz, e, logo, pode ser definido dentro das dimensões de indicadores de recursos (inputs), processos e resultados (outputs) diretos e indiretos.

A Fiocruz adotou um sistema de avaliação programática com atribuição de metas aos seus indicadores bastante interessante, relacionado ao PPA-2011 do MS. No entanto, tal sistema mostrou-se frágil devido à falta de indicadores em dimensões que o modelo proposto nesta pesquisa procurou preencher.

O quadro de indicadores utilizado pela Fiocruz não revelou apenas a ausência de dimensões e subdimensões, como as de recursos informacionais, materiais e espaço físico, organizacionais, de utilização de resultados e dos seus efeitos, mas também mostrou a característica limitada dos indicadores presentes no Relatório Anual de Gestão 2011. É nesse sentido que a presente pesquisa sugere a adoção de novos indicadores, visando a contemplar essas dimensões até então negligenciadas.

Dessa forma, pode-se apontar o modelo proposto em substituição ao quadro de indicadores utilizado pela Fiocruz como uma ferramenta avaliativa mais pertinente, por considerar que a instituição exerce suas atividades de CT\&I relacionando-se com seu ambiente externo e, por consequência, permite à Fiocruz reorientar-se quanto ao seu papel institucional. 


\section{Referências bibliográficas}

BarRÉ, R. The European Perspective on S\&T Indicators. Scientometrics, v. 38, n. 1, p. 57-70, 1997.

BerRy, M. M. J.; TAgGaRT, J. H. Managing technology and innovation: a review. R\&D Management, Oxford, v. 24, n. 4, p. 341-352, oct. 1994.

Brasil. Ministério da Saúde. Fiocruz. Prestação de Contas Ordinária Anual. Relatório de Gestão do Exercício de 2011. Rio de Janeiro: Fiocruz, mar. 2012. Disponível em: < http://www.diplan.fiocruz.br/cgi/cgilua.exe/sys/start.htm?sid=39 >. Acesso em: 6 dez. 2012.

Brasil. Ministério do Planejamento, Orçamento e Gestão (MPOG). Indicadores de programas: Guia Metodológico. Brasília: MP, 2010. Disponível em: <www.planejamento.gov.br>. Acesso em: 6 dez. 2013.

Brisolla, S. N. Indicadores para apoio à tomada de decisão. Ciência da Informação, Brasília, v. 27, n. 2, p. 221-225, maio/ago. 1998. Disponível em: <www.scielo.br/ pdf/ci/v27n2/2729819.pdf>. Acesso em: 6 jun. 2012.

Indicadores de Innovación: Los siete pecados capitales. In: IV Taller Iberoamericano de Indicadores de Ciencia y Tecnología, 1999, México - D.F. Anais... Buenos Aires - Argentina: RICYT, 1999. Disponível em: <www.ricyt.edu.ar/interior/ normalizacion/IV_taller/brisolla.pdf>. Acesso em: 5 jun. 2012.

Clark, A. Evaluation Research. Thousand Oaks/CA: Sage Publications, 1999, p. 14. In: LıMA, N. P. C. Avaliação das Ações de Ciência, Tecnologia e Inovação (CT\&I): Reflexões sobre métodos e práticas. Brasília/DF: CGEE, 2004, p. 6.

Contandriopoulos, A. P. Avaliar a Avaliação. In: Brousselle, A.; Champagne, F.; Contandriopoulos, A. P.; Hartz, Z. Avaliação: conceitos e métodos. Rio de Janeiro: Fiocruz, 2011. Capítulo 12, p. 263-272.

Fundação de Amparo à Pesquisa do Estado de São Paulo. Indicadores de Ciência, Tecnologia e Inovação em São Paulo - 2010. São Paulo: FAPESP, 2010.

HADJI, C. A avaliação, regras do jogo: das intenções aos instrumentos. 4ạ ed. Porto: Porto Editora, 1994.

LiberAL, C. G. Indicadores de ciência e tecnologia: conceitos e elementos históricos: Ciência \& Opinião. Curitiba, v. 2, n. 1/2, jan./dez. 2005. Disponível em: <cienciaeopiniao.up.edu.br/arquivos/cienciaeopiniao/File/volume3/ CienciaOpiniao3_art6.pdf>. Acesso em: 5 jun. 2012.

Moraes, M. F. de; Ohayon, P.; Leitão, D. M. Indicadores de Avaliação no CENPES/ Petrobrás. Revista de Administração, São Paulo, v. 24, n. 2, p. 122-128, abr./ jun. 1989.

Organização para a Cooperação e Desenvolvimento Econômico-OCDE. Medição de atividades científicas e tecnológicas. Manual Frascati. In: Cadernos de Informação em Ciência e Tecnologia/CNPq, João Pessoa, n. 2, p. 15, 1978. 
OHAYon, P. Quadro Metodológico para Implementação de um Sistema de Indicadores de Avaliação na FAPERJ - Fundação de Amparo à Pesquisa do Estado do Rio de Janeiro. In: XVI Simpósio Nacional de Pesquisa de Administração em CT\&I, 1991, Rio de Janeiro/RJ. Anais. São Paulo: FEA-USP, 1991, p. E81-E104. 1 CD-ROM.

Ohayon, P. Modelo Integrado de Indicadores de Ciência, Tecnologia e Inovação no Estado do Rio de Janeiro. Rio de Janeiro: UFRJ, 2007. Projeto de pesquisa, Edital Universal 2004.

Oliveira, M. M. Conhecendo alguns tipos de pesquisa. In: Como fazer pesquisa qualitativa. Rio de Janeiro: Vozes, 2007.

Rockart, J. F. Chief executives define their own data needs. Harward Business Review, Boston, p. 81-92, Mar/Apr 1979. In: MÜLLER, Gisela Regina. Fatores críticos do sucesso de cursos de graduação. 2003. 379f. Tese (Doutor em Engenharia de Produção) - Universidade Federal de Santa Catarina, Florianópolis, 2003. Disponível em: <http://teses.eps.ufsc.br/defesa/pdf/778.pdf>. Acesso em: 24 abr. 2008.

Rubenstein, A. H. Evaluation of Projects and Programs: Before, During and After. In: Managing Technology in the Decentralized Firm. New York: authors Choice Press, 2001, p. 289-342.

Silva E Silva, M. O. (Org.) Avaliação de Políticas e Programas Sociais - Teoria e Prática. São Paulo: Veras, 2001.

VıotTı, E. B.; MACEDo, M. M. Indicadores de ciência, tecnologia e inovação no Brasil. Campinas: Unicamp, 2003.

Worthen, B. R.; SAnders, J. R.; Fitzpatrick, J. L. Avaliação de programas: concepções e práticas. São Paulo: Gente/Edusp, 2004, p. 373-423. Capítulo 15, Como planejar a forma de conduzir uma avaliação.

YIN, R. K. Estudo de caso: planejamento e métodos. Porto Alegre: Bookman, 2005.

Pierre Ohayon

Doutor (com distinção) em Administração de Empresas pela Faculdade de Economia, Administração e Contabilidade da Universidade de São Paulo (USP). Professor da Faculdade de Administração e Ciências Contábeis da Universidade Federal do Rio de Janeiro (UFRJ). Contato: pohayon@facc.ufrj.br

Gerson Rosenberg

Doutor em Tecnologia de Processos Químicos e Bioquímicos pela Escola de Engenharia Química Universidade Federal do Rio de Janeiro (UFRJ). Atua como Tecnologista em Saúde Pública da Fundação Oswaldo Cruz (Fiocruz/ MS). Contato: gerson@fiocruz.br 